\title{
Age-related Changes of the Monoamine Oxidases in Rat Heart
}

\author{
Carlo Cavallotti', Massimo Mancone', Marco Artico ${ }^{2}$ and Daniela Cavallotti ${ }^{3}$ \\ ${ }^{1}$ Department of Cardiovascular and Respiratory Sciences, University of Rome "La Sapienza", via A. Borelli 50, 00161 Rome, ${ }^{2}$ Depart- \\ ment of Pharmacology of Natural Molecules and General Physiology, University of Rome, and 'Service of Neurology, S. Spirito Hospi- \\ tal, Rome, Italy
}

(Received 7 January 2002; and accepted 12 March 2002)

\begin{abstract}
Levels and distribution of heart monoamine oxidases (MAOs) were studied in male SpragueDawley rats of 3 (young) and 24 (old) months of age using biochemical and histochemical techniques. Biochemical assay of MAOs in atria and in ventricles showed a significant increase in MAOs levels in old in comparison with young animals. MAOs histochemical staining revealed an homogeneous distribution of the enzyme within both right and left atria and/or ventricles in 3months-old rats. In 24-months-old rats an increase of MAOs reaction in comparison with younger animals was found. This increase is more consistent in the endocardial portion of the right atrium and in the endocardial portion of the left atrium. In 3-months-old rats MAOs reaction was slightly lower in the epicardial than in the endocardial portions of both atria and/or ventricles. In 24 months-old rats MAOs reaction showed a significant increase if compared with young rats. The significance of these data is discussed in relation to the role of MAOs in rat heart.
\end{abstract}

The monoaminooxidases (MAOs) are intracellular enzymes linked to the mitochondrial membranes. They play an important role in the biogenic amines metabolism (26). The biogenic amines are the catecholamines that, after being released in the synaptic cleft, are toxic for the surrounding structures and, therefore, must be rapidly either reuptaked or metabolised.

MAOs exist in two molecular forms: MAO-A and MAO-B (5), which are different both in specific substrates and inhibitor sensitivity. MAO-A is sensitive to clorgyline while MAO-B is sensitive to deprenyl. Recently a third form of MAOs has been identified in some mammal tissues, the so called MAO-C, which is not sensitive either to clorgyline or deprenyl. This third MAOs form uses benzylamine as substrate and is sensitive to the inhibitor semicarbazide (18).

Correspondence to: Dr. Carlo Cavallotti at above address. Fax: +39-06-4957669

Tel.: +39-06-4958291 E-mail: Cavallotti@uniromal.it
MAOs contribute to the regulation of the cardiac activity by maintaining an unmodified level of catecholaminergic neurotransmitters (8). These enzymes show a sharp age-dependent increase above all in the rat heart, as it is demonstrated by numerous biochemical studies $(1,3,11,15)$. MAOs in rat heart were extensively studied about 30 years ago $(10,11$, 25 ). In recent years these enzymes have come again into fashion owing to their important role as modulator of catecholamines and/or protector versus the toxic action of the free radicals $(2,19,24)$.

In fact, MAOs were restudied in plasma and tissues of mammals (2), new autoradiographic methods were employed in these studies $(21,28)$ and a kinetic characterization of these enzymes was made in the rat heart (8).

Species differences were described in age related changes of MAOs (24) and many drugs were used to modulate and/or to inhibit MAO activities (19). Despite all these studies information about the topographical localization of MAOs in the rat heart is lacking, except for two studies that demonstrated an 
increase of the MAO in old rat heart by means of histochemical techniques $(5,13)$. However these data, according to our experience, show two limitations: 1) these studies consider 14-month-old rats as old and 2) authors are not well-defined concerning the different localization of the MAO in the various heart structures. For this reason both the localization and variations of MAOs in the cardiac tissues of rats were studied by us. Preliminary results obtained in our laboratories were published elsewhere (17).

\section{MATERIALS AND METHODS}

Eight 3-month-old (young) and eight 24-month-old (old) Sprague-Dawley rats were used in this experiment. The animals were kept at a constant temperature $\left(22^{\circ} \mathrm{C}\right)$ with a $10^{-14} \mathrm{~h}$ dark-light rhythm, with free access to water and food. The maximum lifespan in our colony was $26 / 28$ months. Each animal was weighed every day and the data were recorded. The animals were treated according to the Convention of Helsinki on utilization of animals in biomedical research. They were sacrificed by means of terminal anaesthesia, (by an endoperitoneal injection $50 \mathrm{mg} / \mathrm{Kg}$ body weight of Nembutal).

The thoracic cavity was opened and the heart removed, washed, weighed and put into a cold phosphate buffer-solution. The cardiac cavities were identified and portions of the wall of the single cavities were taken, weighed and or homogenized for biochemical dosages, or soaked in cold isopentane, frozen in liquid nitrogen and cut in a cryostat into 10 $-15 \mu \mathrm{m}$ slices for the histochemical staining. A weak postfixation was then accomplished and the section were stained and treated for the observation at the light microscope according to the standard methodologies. The "boundary" - and "dubious" - portions were not utilized. The histochemical staining for the detection of the MAOs was performed according to the method suggested by Uchida and Koelle (27). This method is based on a first preincubation of the sections in a $30 \% \mathrm{Na}_{2} \mathrm{SO}_{4}$ solution for $1 \mathrm{~h}$ at $37^{\circ} \mathrm{C}$. A second preincubation was made in $0.05 \mathrm{M}$ phosphate buffer-solution ( $\mathrm{pH} 7.6$ ) containing sucrose $(8 \%)$ either with or without $10^{-7} \mathrm{M}$ of clorgyline as MAO-A specific inhibitor (12) or deprenyl as MAO-B specific inhibitor (5). The sections were washed in phosphate buffer-solution and stained by incubating them for the third time in a solution containing phosphate buffer, nitroblue tetrazolium, sucrose and tryptamine at $37^{\circ} \mathrm{C}$ for $1-2 \mathrm{~h}$. Then the sections were washed in $\mathrm{H}_{2} \mathrm{O}$, fixed by means of a $10 \%$ formalin solution, dehydrated and mounted in Entellan (Merck). The specificity of the reaction was evaluated by incubating some control sections in a medium without substrate (tryptamine) or without dye (nitroblue tetrazolium). In both cases the reaction was negative. On the contrary, the reaction was positive in the presence of nitroblue tetrazolium precipitates. The granules appear blue on the slide and black on a black-and-white photograph.

Careful examination of samples was made at the same magnification and the same lighting using a Zeiss II photomicroscope and Ilford black-and-white Pan-F films. The results were shown in Table 1 and 2 because of the large number of slides whose analysis should be summarized considering the mean intensity of the reaction in different areas of the various slides. The quantitative analysis of images allows to obtain data regarding many morphological parameters (e.g.: number of cells, surface of a cell, intensity of a reaction, specific parameters, specific surfaces, white or black or grey intensity, etc.). The quantitative analysis of images may be performed by the Quantimet Leica 500 image-analyser. The value 0 , which indicates the negative reaction, may be obtained, as just said, by omitting the substrate in the incubation medium or the specific dye, but also by adding the specific reaction-inhibitor. The value 100 indicates the maximum intensity of the reaction present in each sample. The final values are expressed in Conventional Units (CU士 $\pm_{\text {S.E.M. }}$ ) directly provided by the instrument.

QAI may provide inaccurate results. In fact, the main choices (i.e. the instructions for software) are ordered by each research-worker, according to personal preferences. For these reasons the data are more sham than fair and it is necessary to follow very careful rules. The counts must be repeated at least three times using the technique of the double masked. All the counts should be performed by different research-workers, on different analysers, and with samples identified only by a number or by a letter. Final results must be obtained by another research-worker, who examines experimental protocols to identify each sample and attribute specific values. Final values must be submitted to statistical analysis of data. The values reported represent the intensity of staining for each type of treatment. All these details are reported in the Manual of Methods of the Quantimet Leica 500 image analyser (9).

Amount of protein. In all our experiments, samples of cardiac tissues were weighed and homogenised into an ice-cold homogenisation buffer 1:10 weight/volume. Tissue protein concentrations were 
determined following the method of Lowry (16) using bovine serum albumin (BSA) as standard and Folin phenol as reagent.

Biochemical dosage of MAOs. MAO enzymatic activities were determined on homogenised tissues (1:10 weight/volume) according to Lowe and coworkers (15), all the results are expressed as International Units (nMoles of substrate hydrolysed/mg protein). In brief homogenised samples of various parts of the hearts were tested for changes in MAO activities and two different substrates (kynuramine and tryptamine) were used to distinguish MAOs from other mitochondrial oxidative enzymes, as before suggested by Horita (10).

Statistical analysis. The statistical methods used throughout this study must be interpreted as an accurate description of the data rather than a statistical inference of such data. Preliminary studies of each value were performed using basic sample statistics. Mean values, maximum and minimum limits, variations, standard deviation (S.D.), standard error of the mean (S.E.M.) and correlation coefficients were performed according to Serio (22). The relationship between each group of age was studied using the respective correlation coefficients. Finally, a correlative analysis of the morphological and biochemical data was performed. Correlation coefficients denote a significant level less than $0.001(P<0.001)$. This correlation coefficient was calculated according to Castino and Roletto (4).

\section{RESULTS}

Our results are shown in the tables 1 and 2. Table 1 shows the data of the quantitative analysis of the images in the different heart portions. It is possible to affirm that: 1) there is a decreasing gradient of MAO activity from left ventricle $>$ right ventricle $>$ left atrium > right atrium; 2) in old rats the values are higher than those observed in young rats; and 3) in old rats the MAO activity is prevalently localized in the endocardium.

Table 2 reports some biochemical and numerical values in young and old rats. The biochemical values of the MAO level confirm the corresponding histochemical results. It is also possible to notice that the addition of the inhibitor clorgyline produces the disappearance of the MAO activity, while the use of inhibitor deprenyl causes only a moderate decrease of the MAO activity.

Our results demonstrated that: 1) in young rats
Table 1 MAO activity values in the indicated heart regions

\begin{tabular}{lcc}
\hline Region & young rats $\mathbf{n}=\mathbf{8}$ & old rats $\mathbf{n}=\mathbf{8}$ \\
\hline Left & $45.6 \pm 2.1$ & $76.2 \pm 8.1 *$ \\
ventricle & $10.7 \pm 1.2$ & $23.0 \pm 6.3 *$ \\
Epicardium & $21.9 \pm 1.9$ & $32.9 \pm 7.0 *$ \\
Myocardium & $11.0 \pm 1.7$ & $14.2 \pm 5.0 \mathrm{n.s}$ \\
Endocardium & $32.9 \pm 2.8$ & $89.3 \pm 5.1 *$ \\
Right ventricle & $7.9 \pm 0.9$ & $32.6 \pm 3.4 *$ \\
Epicardium & $14.5 \pm 0.7$ & $23.9 \pm 2.6 *$ \\
Myocardium & $8.3 \pm 0.5$ & $34.1 \pm 4.1 *$ \\
Endocardium & $26.5 \pm 1.9$ & $71.3 \pm 5.6 *$ \\
Left atrium & $11.1 \pm 1.1$ & $29.8 \pm 2.8 *$ \\
Epicardium & $16.9 \pm 1.3$ & $42.4 \pm 3.1 *$ \\
Endocardium & $19.4 \pm 1.6$ & $58.2 \pm 4.3 *$ \\
Right atrium & $9.3 \pm 0.7$ & $16.4 \pm 2.1 *$ \\
Epicardium & $10.8 \pm 1.1$ & $38.3 \pm 3.3 *$ \\
Endocardium &
\end{tabular}

The values are expressed in Conventional Units (C.U. \pm S.E.M.) as explained in the methods. $* P<0.001$ in old versus young.

n.s. $=$ not significant.

Table 2 Weight, numerical and biochemical values in old and young rats

\begin{tabular}{|c|c|c|}
\hline & young rats $n=8$ & old rats $n=8$ \\
\hline $\begin{array}{l}\text { body weight } \\
\text { g. } \pm \text { s.E.M. }\end{array}$ & $240 \pm 15$ & $465 \pm 21^{*}$ \\
\hline $\begin{array}{l}\text { heart weight } \\
\text { g. } \pm \text { s.E.M. }\end{array}$ & $1.46 \pm 0.1$ & $3.30 \pm 0.2 *$ \\
\hline $\begin{array}{l}\text { ratio } \\
\text { heart/body } \\
\text { weight }\end{array}$ & $6.08 \pm 0,11$ & $7.19 \pm 0.23 *$ \\
\hline $\begin{array}{l}\text { MAO activity } \\
\text { left } \\
\text { ventricle** }\end{array}$ & $89.4 \pm 1.3$ & $210.3 \pm 3.2^{*}$ \\
\hline $\begin{array}{l}\text { MAO activity } \\
\text { right } \\
\text { ventricle** }\end{array}$ & $76.2 \pm 1.1$ & $156.5 \pm 4.4^{*}$ \\
\hline $\begin{array}{l}\text { MAO activity } \\
\text { left atrium** }\end{array}$ & $49.4 \pm 1.3$ & $118.2 \pm 2.8^{*}$ \\
\hline $\begin{array}{l}\text { MAO activity } \\
\text { right atrium** }\end{array}$ & $38.5 \pm 1.6$ & $92.4 \pm 2.1^{*}$ \\
\hline $\begin{array}{l}\text { left ventricle }+ \\
\text { clorgiline } 10^{-7} \mathrm{M}\end{array}$ & - & - \\
\hline $\begin{array}{l}\text { left ventricle }+ \\
\text { deprenyl } 10^{-7} \mathrm{M}\end{array}$ & $29.5 \pm 1.6$ & $71.3 \pm 2.0^{*}$ \\
\hline \multicolumn{3}{|c|}{$\begin{array}{l}\text { Average values found in single animals } \pm \text { s.E.M. } \\
N=\text { number of animals } \\
* P<0.001 \text { in old versus young. } \\
* * \text { International units (I.U.) } \pm \text { S.E.M. (see Methods) }\end{array}$} \\
\hline
\end{tabular}


the MAO shows a more intense activity in the median portion of the left ventricle of the heart, whereas the same activity is equal in the subendocardial and subepicardic portions. On the contrary, in the right ventricle the MAO activity is uniformly distributed in all the thickness of the wall; 2) in old rats the MAO activity is more intense than in young rats. The old rats' left ventricle shows a more intense MAO activity with the same distribution of that observed in young rats. The most relevant increase of the MAO reaction can be observed in the right ventricle. 3) Clorgyline is a specific MAO inhibitor and, if added to the reaction, induces the disappearance of the MAO activity, whereas the deprenyl is a partial inhibitor and, if added to the reaction, causes only a moderate decrease of the MAO activity.

\section{DISCUSSION}

Our findings were obtained by the use of histochemistry and confirmed by biochemical assay. Generally, histochemical findings are requested to be confirmed also by other techniques, including light auto-radiography with selective markers, immunohistochemistry and /or in situ hybridation. Many of these findings are in progress in our laboratories. Now, therefore, we write this paper as communication, without figures of histochemistry, but summarizing in tables our findings.

The monoaminooxidases are enzymes that regulate the catecholaminergic neurotransmitters levels and, since they have an important role in the control of the cardiac activity, it is possible to understand why the anti-MAO drugs are utilized in the control of some cardiac diseases $(6,14)$. Amine oxidase activities may also be involved in the regulation of the response to some changes in isolated cardiomyocytes of spontaneously hypertensive rats (18) and of the phenylephrine kinetics in isolated rat heart (20). The role of MAO activities may be larger and more important in the light of recent studies. In fact, Fischer et al. (7) have proven that 5-hydroxytryptamine stimulates glucose transport in cardiomyocytes via a monoamine oxidase-dependent reaction. Moreover, Lamontagne et al. (14) focused the attention on the importance of monoamine oxidation in the control of tissue noradrenaline content in isolated perfused rat heart during ischemia. The usefulness of an antioxidant strategy related to modifications of endogenous antioxidant enzymes during heart ischemia and of a possible pharmacological modulation of free-radical generation and peroxidation of lipids (with relevant consequences on moni- toring of tissue damage) may result in a significant amelioration in the therapy of acute ischemic conditions (6). In fact, the role of monoaminoxidase in the intensification of peroxidation of lipids in mitochondria during experimental myocardial necrosis was already demonstrated (14). Moreover, the significant role of these enzymes in the metabolic pathways of different organs as well as in various pathological conditions could be related to their changes and their alterations with age.

In fact, MAOs hyperactivity (being the enzymes associated to the mitochondrial membranes) produces a significant increase in the development of oxygen free radicals. The possible mechanisms of cell damage induced by free radicals include (23): 1) reactions with nucleic acids, nucleotides, polysaccharides, protein and non-protein thiols (oxidation of thiols); 2) covalent bond with the components of membranes (lipids, proteins, enzymes, receptors and transport systems); and 3) triggering of the lipid peroxidation reaction.

The oxidative stress due to the action of the free radicals is cytotoxic and may be inhibited by inhibitors of MAO-B as L-Deprenyl.

This role should further be investigated by experimental and clinical studies focusing the attention on possible different age-dependent effects and mechanisms of MAO activities.

\section{ACKNOWLEDGEMENTS}

This study was supported by grants from University of Rome "La Sapienza" (Dip. 01.043.14). The authors are greatly indebted to Drs. B. Nagar and V. Malinovska for their suggestions and criticism. The Medline, Internet and other informatic consulting services of Drs. M. Cameroni and F.M. Tranquilli Leali are gratefully acknowledged. The photographic service of Mr. G. Leoncini, the secretarial work of Mrs. S. Casamento and the kind help of Mrs. S. Hobby in the revision of the English language are also gratefully acknowledged.

\section{REFERENCES}

1. Blascliko H. (1974) The natural history of amine oxidase. Rev. Physiol. Biochem.Pharmacol. 70, 112-196.

2. Boomsma F., van Dijk J., Bhaggoe UM., Bouhuizen AM., van den Meiracker AH. (2000) Variation in semicarbazidesensitive amine oxidase activity in plasma and tissues of mammals. Comp. Biochem. Physiol. Toxicol. Pharmacol. 126, 69-78.

3. Cao Danh H., Strolin Benedetti M., Dostert P. and Mousset A. (1984) Age-related changes in benzylamine oxidase activity in rat tissues. J. Pharm. Pharmacol. 36, 592-596. 
4. Castino M. Roletto E. (1992) Statistica Applicata. Edizioni Piccin Padova.

5. Cawthon R. M. and Breakfield X. O. (1979) Differences in A and $\mathrm{B}$ forms of monoamineoxidase revealed by limited proteolysis and peptide mapping. Nature. 281, 692-694.

6. Dzhafarov A. I., Magomedov N. M., Azimova A. M., Alieva N. I., Dagkesa-manskaia D. N. (1988).. Role of monoaminoxidase in the intensification of peroxidation of lipids in mitochondria during experimental myocardial necrosis. Biull. Eksp. Biol. Med. 106, 45-47.

7. Fischer Y., Thomas J., Kamp J., Jungling E., Rose H., Carpene C., Kammermeier H. (1995) 5-hydroxytryptamine stimulates glucose transport in cardiomyocytes via a monoamine oxidase-dependent reaction. Biochem. J. 311, 575 -583 .

8. Guimaraes JT., Vindis C., Soares-da-Silva P., Parini A. (1988) Molecular and kinetic characterization of monoamine oxidases in the rat heart. Biochem. Soc. Trans. 26, S 392.

9. Handbook of Methods Quantimet 500 Leica Microsystems Imaging Solutions (1997) Clifton Road, Cambridge, U.K.

10. Horita A. (1967) Cardiac monoamine oxidase in rat. Nature. 215, 411-412.

11. Horita A. (1968) The influence of age on the recovery of cardiac monoamine oxidase after irreversible inhibition. Biochem. Pharmacol. 17, 2091-2096.

12. Knoll J. and Magyar K. (1972) Some puzzling pharmacological effects of monoamine oxidase inhibitors.dv. Biochem. Psychopharmacol. 5, 393-408.

13. Lai F. M., Berkowitz B., and Spector. (1978) Influence of age on brain vascular and cardiovascular monoamine oxidase activity in the rat. Life Sci. 22, 2051-2056.

14. Lamontagne D., Yamaguchi N., Ribuot C. (1991) de Champlain J., Nadeau R. Reduction of tissue noradrenaline content in the isolated perfused rat heart during ischemia: importance of monoamine oxidation. Can. J. Physiol. Pharmacol. 69, 1190-1195.

15. Lowe M. C., Reichenbach D. and Horita A. (1975) Extraneuronal monoamine oxidase in rat heart: biochemical characterization and electron microscopic localization. $J$. Pharmacol. Exp. Ther: 194, 522-536.

16. Lowry O. H., Rosebrough N. J., Farr A. L. and Randall J. (1951) Protein measurement with the folin phenol reagent. $J$. Biol. Chem. 193, 265-275.
17. Meco M., Bonifati V., Collier W. L., Ramacci M. T., Amenta F. (1987) Enzyme Histochemistry of Monoamine oxidase in the Heart of Rats. Mechanisms of Ageing and Development. $28,145-155$.

18. Pino R., Failli P., Mazzetti L., Buffoni F. (1997) Monoamine oxidase and semicarbazide-sensitive amine oxidase activities in isolated cardiomyocytes of spontaneously hypertensive rats. Biochem. Mol. Med. 62, 188-196.

19. Raasch W., Muhle H., Dominiak P. (1999) Modulation of MAO activity by imidazoline and guanidine derivatives. Ann. N. Y. Acad. Sci. 881, 313-331.

20. Raffel D. M., Wieland D. M. (1999) Influence of vesicular storage and monoamine oxidase activity on $\mathrm{C}^{11}$ phenylephrine kinetics: studies in isolated rat heart. J. Nucl. Med. 40, 323330.

21. Saura J., Kettler R., Da Prada M., Richards JG (1992) Quantitative enzyme radioautography with $3 \mathrm{H}-\mathrm{Ro} 41-1049$ and $3 \mathrm{H}-$ Ro 19-6327 in vitro: localization and abundance of MAO-A and MAO-B in rat CNS, peripheral organs, and human brain. J. Neurosci 12, 1977-1999.

22. Serio A. (1986) Appunti delle lezioni di Statistica Sanitaria. Ed. Kappa Roma.

23. Slater T.F. (1984) Free-radical mechanisms in tissue injury. Biochem. J. 222, 1-15.

24. Strolin Benedetti M., Thomassin J., Tocchetti P., Dostert P., Kettler R., Da Prada M. (1994) Species differences in changes of heart monoamine oxidase activities with age. $J$. Neural. Transm. 41, 83-87.

25. Studer A., Baumgartner H. R. and Reber K. (1964) Histochemical evidence of monoamine oxidase activity in rats of different ages. Histochemie. 4, 43-47.

26. Tipton K. F., Houslay M. D. and Mantle T. J. (1976) The nature and location of the multiple forms of monoamine oxidase, in Monoamineoxidase and its inhibition. CIBA Found. Symp. 39, 5-25.

27. Uchida E. and Koelle G. B. (1984) Histochemical investigation of criteria for the distinction between monoamine oxidase $\mathrm{A}$ and $\mathrm{B}$ in various species. J. Histochem. Cytochem. 32, 667-673.

28. Waldmeier PC., Stocklin K. (1990) Binding of [3H] brofaromine to monoamine oxidase A in vivo: displacement by clorgyline and moclobide. Eur: J. Pharmacol. 180, 297-304. 